\title{
The pulsations of the B5IVe star HD 181231 observed with CoRoT and ground-based spectroscopy ${ }^{\star}, \star \star$
}

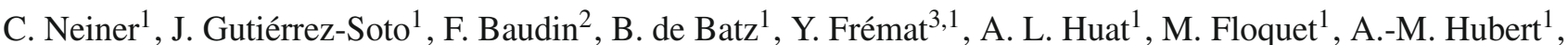
B. Leroy ${ }^{4}$, P. D. Diago ${ }^{5}$, E. Poretti ${ }^{6}$, F. Carrier ${ }^{7}$, M. Rainer ${ }^{6}$, C. Catala ${ }^{4}$, O. Thizy ${ }^{8}$, C. Buil ${ }^{9}$, J. Ribeiro ${ }^{10}$, L. Andrade ${ }^{11}$, M. Emilio ${ }^{12}$, F. Espinosa Lara ${ }^{1}$, J. Fabregat ${ }^{5}$, E. Janot-Pacheco ${ }^{11}$, C. Martayan ${ }^{3,1}$, T. Semaan ${ }^{1}$, J. Suso ${ }^{5}$, A. Baglin ${ }^{4}$, E. Michel ${ }^{4}$, and R. Samadi ${ }^{4}$

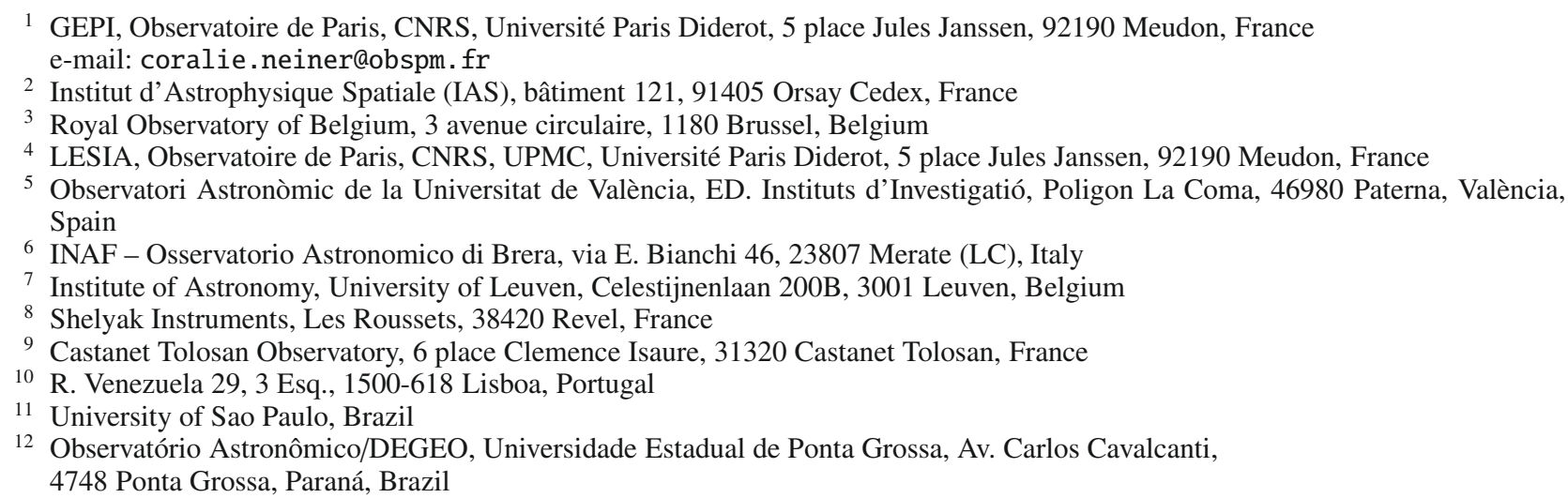

Received 28 February 2009 / Accepted 16 July 2009

\section{ABSTRACT}

\begin{abstract}
Context. HD 181231 is a B5IVe star, which has been observed with the CoRoT satellite during $\sim 5$ consecutive months and simultaneously from the ground in spectroscopy and spectropolarimetry.

Aims. By analysing these data, we aim to detect and characterize as many pulsation frequencies as possible, to search for the presence of beating effects possibly at the origin of the Be phenomenon. Our results will also provide a basis for seismic modelling. Methods. The fundamental parameters of the star are determined from spectral fitting and from the study of the circumstellar emission. The CoRoT photometric data and ground-based spectroscopy are analysed using several Fourier techniques: CLEAN-NG, PASPER, and TISAFT, as well as a time-frequency technique. A search for a magnetic field is performed by applying the LSD technique to the spectropolarimetric data.

Results. We find that HD 181231 is a B5IVe star seen with an inclination of $\sim 45$ degrees. No magnetic field is detected in its photosphere. We detect at least 10 independent significant frequencies of variations among the 54 detected frequencies, interpreted in terms of non-radial pulsation modes and rotation. Two longer-term variations are also detected: one at $\sim 14$ days resulting from a beating effect between the two main frequencies of short-term variations, the other at $\sim 116$ days due either to a beating of frequencies or to a zonal pulsation mode.

Conclusions. Our analysis of the CoRoT light curve and ground-based spectroscopic data of HD 181231 has led to the determination of the fundamental and pulsational parameters of the star, including beating effects. This will allow a precise seismic modelling of this star.
\end{abstract}

Key words. stars: early-type - stars: emission-line, Be - stars: individual: HD 181231 - stars: rotation - stars: oscillations

\section{Introduction}

Be stars are main sequence or slightly evolved non-supergiant, late-O, B, or early-A stars that show or have shown at least once

\footnotetext{
* Based on observations obtained with the CoRoT satellite, with FEROS at the $2.2 \mathrm{~m}$ télescope of the La Silla Observatory under the ESO Large Programme LP178.D-0361, with Narval at the Télescope Bernard Lyot of the Pic du Midi Observatory, and collected from the BeSS database.

$\star \star$ Table 5 is only available in electronic form at the CDS via anonymous ftp to cdsarc.u-strasbg.fr $(130.79 .128 .5)$ or via http://cdsweb.u-strasbg.fr/cgi-bin/qcat? J/A+A/506/143
}

emission in their Balmer lines caused by the presence of a decretion circumstellar disk. Emission can also appear in other lines of the spectrum, in particular in red HeI lines or FeII lines. Moreover, the disk produces an infrared excess in the spectral energy distribution. In addition to this disk, Be stars also exhibit polar winds. About $20 \%$ of all B stars in our galaxy are Be stars. However, this fraction depends on metallicity (Martayan et al. 2006, 2007). Therefore, in other environments of lower metallicity, the fraction of Be stars over B stars can be much higher. Only B stars of sufficiently high rotational velocity at the ZAMS 
Table 1. Log of the spectroscopic and spectropolarimetric observations.

\begin{tabular}{llll|llll}
\hline \hline Instr. & Date & $T_{\exp }(\mathrm{s})$ & $\#$ & Instr. & Date & $T_{\exp }(\mathrm{s})$ & $\#$ \\
\hline BeSS & 25.05 .07 & 4500 & 1 & FEROS & 01.07 .07 & $600-660$ & 6 \\
BeSS & 03.06 .07 & 5531 & 1 & FEROS & 02.07 .07 & $510-770$ & 6 \\
BeSS & 08.06 .07 & 6900 & 1 & Narval & 02.07 .07 & $4 * 900$ & 2 \\
BeSS & 23.06 .07 & 5430 & 1 & FEROS & 03.07 .07 & $550-630$ & 5 \\
Narval & 23.06 .07 & $4 * 900$ & 2 & FEROS & 04.07 .07 & $630-750$ & 4 \\
FEROS & 25.06 .07 & 900 & 4 & Narval & 04.07 .07 & $4 * 900$ & 2 \\
Narval & 25.06 .07 & $4 * 900$ & 2 & BeSS & 07.07 .07 & 1800 & 1 \\
FEROS & 26.06 .07 & 900 & 2 & BeSS & 12.07 .07 & 7800 & 1 \\
Narval & 26.06 .07 & $4 * 900$ & 2 & BeSS & 13.07 .07 & 3618 & 1 \\
FEROS & 27.06 .07 & 900 & 4 & FEROS & 16.07 .07 & 900 & 6 \\
Narval & 27.06 .07 & $4 * 900$ & 2 & FEROS & 17.07 .07 & 900 & 6 \\
FEROS & 28.06 .07 & $550-870$ & 5 & FEROS & 18.07 .07 & $800-900$ & 5 \\
Narval & 28.06 .07 & $4 * 900$ & 2 & FEROS & 19.07 .07 & $700-900$ & 5 \\
FEROS & 29.06 .07 & $810-850$ & 4 & FEROS & 20.07 .07 & $600-900$ & 4 \\
Narval & 29.06 .07 & $4 * 900$ & 2 & BeSS & 21.07 .07 & 4222 & 1 \\
FEROS & 30.06 .07 & 900 & 6 & BeSS & 10.10 .07 & 4500 & 1 \\
Narval & 30.06 .07 & $4 * 900$ & 2 & & & & \\
\hline
\end{tabular}

The origin of the spectra, date, exposure time, and number of spectra acquired on that date are provided.

can become Be stars (Martayan et al. 2007), and this velocity depends on the metallicity of the protostellar environment.

Although Be stars are known to be fast rotators (typically $v \sin i=250 \mathrm{~km} \mathrm{~s}^{-1}$ ), their velocities are not high enough to reach the critical limit at which the centrifugal force compensates gravity. Indeed, Be stars rotate on average at $88 \%$ of the critical angular velocity in our galaxy (Frémat et al. 2005), and Cranmer (2005) found significantly subcritical rotation speeds for early-type Be stars. Thus, at least in most cases, rotation by itself cannot explain the ejection of matter from the star that leads to the formation of the decretion disk. Several explanations can be put forward to provide additional angular momentum and reach the critical limit, e.g., the interaction with a binary companion, the beating of non-radial pulsation modes, or the presence of a magnetic field.

Light and line-profile short-term variability due to non-radial pulsations and rotational modulation is present in the quasitotality $(86 \%)$ of early-Be stars, in $40 \%$ of mid-types (B4e-5e) and in only $18 \%$ of late-Be stars (Hubert \& Floquet 1998). Variability on other timescales is also present. For a complete review of Be stars, we refer to Porter \& Rivinius (2003).

The driving mechanism of pulsations in Be stars is the $\kappa$ mechanism, i.e., an effect of the opacity of iron-peak elements in the envelope of the star (Dziembowski \& Pamiatnykh 1993; Dziembowski et al. 1993). This mechanism only occurs when the opacity bump coincides with the adiabatic and non-adiabatic transition zone. Pressure $(\mathrm{p})$ and gravity $(\mathrm{g})$ modes can then be auto-excited, depending on the depth at which the iron opacity bump is situated in the envelope. Modes trapped near avoided crossings can also be observed.

In this paper, we describe the observations of the B5IVe star HD 181231 obtained with CoRoT and ground-based spectroscopy (Sect. 2) and the characteristics of the star (Sect. 3). We present the results of a time-series analysis performed on these data (Sect. 4) and discuss them in terms of pulsations and rotation (Sect. 5). Finally, we present our conclusions in Sect. 6.

HD 181231 is a B5IVe star that exhibits clear variations in its CoRoT photometric light curve. According to the determination of the stellar fundamental parameters by spectral fitting and the shape of the emission lines from the disk, the inclination of the star is $\sim 45$ degrees.
No longitudinal magnetic field is detected down to a level of $\sim 650 \mathrm{G}$. Using an inclination angle of $i=45$ degrees, this corresponds to a maximum oblique dipole effective field of $B_{\text {eff }}=3200 \mathrm{G}$.

\section{Observations}

The star HD 181231 was observed with the CoRoT satellite for $\sim 5$ consecutive months. Ground-based observations were simultaneously obtained for about three weeks in terms of spectroscopy with FEROS mounted on the ESO $2.2 \mathrm{~m}$ telescope and in terms of spectropolarimetry with Narval mounted on the TBL (Pic du Midi, France). Additional spectra from the BeSS database were also collected. A log of the observations is provided in Table 1.

\subsection{CoRoT photometric observations}

CoRoT was launched on December 27, 2006. The raw photometric data acquired with CoRoT were reduced by the CoRoT team. The corrections applied to the data were the subtraction of the offset and background, the correction for the cross-talk effect, the transformation from ADU to electrons, the corrections of the exposure time variations, jitter effect, and outliers. For more details, we refer to Auvergne et al. (2009) and Samadi et al. (2006).

In the asteroseismology field, CoRoT obtains one measurement every second. Averaged measurements every $32 \mathrm{~s}$ are then calculated and constitute the light curve, with time converted to the heliocentric system. A flag is assigned to each datapoint to indicate if this measurement has been obtained during a proton impact, the South Atlantic Anomaly (SAA) crossing, a transition of the satellite from light to penombra or vice versa, a large jitter correction, or a light-curve discontinuity because of temperature effects.

In this paper, we analysed both the light curve with only datapoints with flag 0 , i.e., taken in perfect conditions, which corresponds to 377298 photometric measurements, and the light curve for which the 45059 points with non-0 flag have been replaced by interpolated points or uncorrected.

HD 181231 was observed by CoRoT during its LRC1 long run. The observations lasted 156.64 days from May 11 to October 15, 2007, and were uninterrupted. The CoRoT light curve (Fig. 1) was detrended with a linear fit to remove the linear effect of ageing of some instrument parts (CCDs and/or optics). The light curve shows variations with a maximum peak-to-peak amplitude of 11 millimag (see top panel of Fig. 1) and a peakto-peak point scatter due to noise of less than 0.8 millimag. The noise level of the CoRoT data for HD 181231 is about 3 ppm in 5 days, i.e., very close to the photon noise of $2 \mathrm{ppm}$. The difference is caused by a jitter correction residue.

\subsection{FEROS spectroscopic observations}

HD 181231 was observed from June 26 to July 21, 2007, at ESO/La Silla with the FEROS spectrograph attached to the $2.2 \mathrm{~m}$ telescope. Unfortunately, the dates of our FEROS observations coincide with the minimum amplitude of variations in the CoRoT light curve (see Figs. 1 and 6).

A total of 72 échelle spectra were obtained with exposure times of between 510 and $900 \mathrm{~s}$ and a signal to noise of 140 to 250. The spectral resolution is 48000 and the wavelength ranges from 3500 to $9200 \AA$ on 39 échelle orders. This observing time was awarded in the framework of an ESO Large Programme 


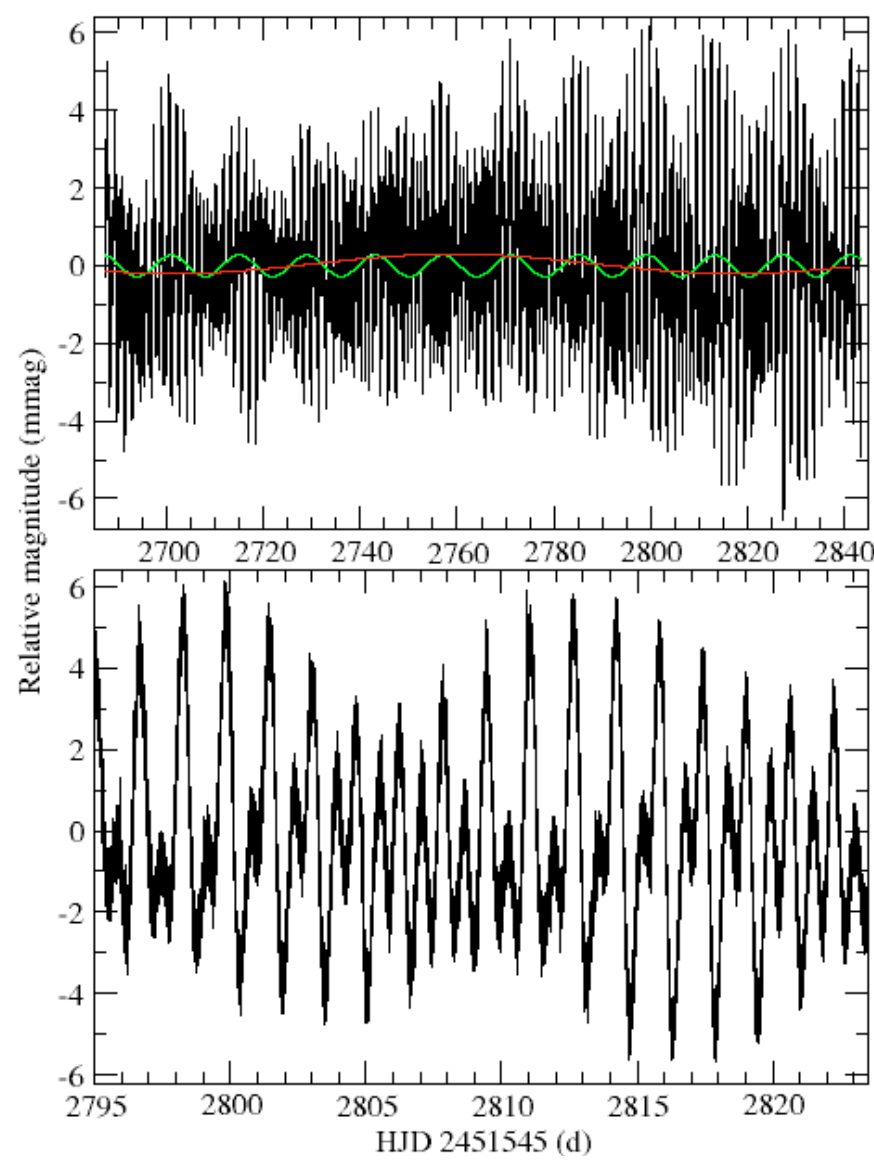

Fig. 1. Full 156-day (top) and zoom (bottom) of the CoRoT light curve of HD 181231, detrended with a linear fit. On the top panel two sine fits are overimposed, which correspond to a variation with 116.5 and 14.1 days.

(LP178.D-0361; PI: Poretti) dedicated to CoRoT asteroseismic targets.

We reduced the FEROS spectra using an improved version of the standard FEROS pipeline, written in MIDAS, developed by Rainer (2003). For more details, we refer to Uytterhoeven et al. (2008). The spectra were corrected for heliocentric velocity.

\subsection{Narval spectropolarimetric observations}

A set of spectropolarimetric observations was obtained with Narval mounted on the 2-m Télescope Bernard Lyot (TBL) at Pic du Midi (France) from June 23 to July 4, 2007 (PI: Catala). The Narval observations were collected at the same time as the FEROS observations, i.e., at the minimum amplitude of variations in the CoRoT light curve (see Figs. 1 and 6).

Eighteen échelle spectra were acquired with signal-to-noise ratio of between 110 and 190. The spectral resolution is 65000 and the wavelength ranges from 3690 and $10480 \AA$. They have been obtained in spectropolarimetric mode in Stokes $V$ sequences of $4 * 900 \mathrm{~s}$ each.

The reduction of the spectra was performed using the LibreEsprit pipeline (based on Esprit, Donati et al. 1997) at the telescope by the TBL team. The spectra were corrected for heliocentric velocity. The 18 intensity spectra extracted from each polarimetric sequence are used in the spectral analysis in this paper, rather than the individual exposures, because they have higher
Table 2. Apparent value of the stellar parameters of HD 181231.

\begin{tabular}{lll}
\hline \hline & F06 & This work \\
\hline$T_{\text {eff }}(\mathrm{K})$ & $14000 \pm 1000$ & $13700 \pm 1500$ \\
$\log g$ & $3.73 \pm 0.10$ & $3.47 \pm 0.20$ \\
$v \sin i$ spectral fit $\left(\mathrm{km} \mathrm{s}^{-1}\right)$ & $250 \pm 30$ & $175 \pm 25$ \\
$v \sin i$ Fourier $\left(\mathrm{km} \mathrm{s}^{-1}\right)$ & & $165 \pm 30$ \\
\hline
\end{tabular}

signal-to-noise ratio. The corresponding 18 Stokes $V$ spectra are used in the search for a magnetic field.

\subsection{BeSS spectra}

Nine $\mathrm{H} \alpha$ spectra obtained during the CoRoT run by amateur astronomers were retrieved from the BeSS database ${ }^{1}$ and are used in this study as an indication of the emission level and variability of the star. All these spectra were collected with Lhires III spectrographs ${ }^{2}$ with gratings of either 2400 or 1200 lines/mm on telescopes of diameters of between 23 and $36 \mathrm{~cm}$. The typical resolution of these spectra is $\sim 15000$.

\section{The star HD 181231}

\subsection{Fundamental parameters}

HD 181231 is a B5IVe star (Frémat et al. 2006, hereafter F06) of magnitude $V=8.58$. According to the Hipparcos catalogue (Perryman et al. 1997), it is situated at $3.36 \mathrm{kpc}$, but at only $590 \mathrm{pc}$, according to Kozok (1985).

In F06, we determined the fundamental parameters of HD181231 from one spectrum extracted from the GAUDI database, obtained with the Cassegrain spectrograph mounted on the Boller \& Chivens telescope in Brazil with a resolution of $\sim 7000$ and a signal-to-noise ratio of 80 . The parameters found in F06 are reported in Table 2.

By analysing the new spectra acquired for this work, which are of both higher spectral resolution and higher signal-to-noise ratio, we can re-determine the parameters with a higher confidence level. We used an average of the 72 FEROS spectra. The new data clearly show that, in spite of the low effective temperature, the spectrum of HD 181231 exhibits a large number of small emission features superimposed on photospheric lines that mainly belong to $\mathrm{HI}$, Fe II, Si II, C II, and Mg II. The presence of these features (invisible in the lower resolution 2006 data) makes the continuum normalization and the interpretation of the spectrum far more complex than assumed in F06.

In Fig. 2, we compare the 2006 data (black) of F06 with the average of the new time series data (red) that we convolved with a Gaussian profile to achieve a comparable, lower resolution. Differences between the 2 sets of spectra can be seen in some spectral lines (He I 4026 and 4471, Mg II 4481), due to emission and possibly to pulsations, while the hydrogen lines seem to be slightly broader in the 2006 spectrum.

The effective temperature $\left(T_{\text {eff }}\right)$, surface gravity $(\log g)$, and projected rotation velocity $(v \sin i)$ are derived from the average FEROS spectrum using the procedure described in F06. We assume a solar metallicity. Our main fitting criteria are the neutral helium lines and the wings of the $\mathrm{H} \delta$ line, which exhibits a weaker emission core. The wavelength range 4400-4450 $\AA$ is ignored in the fitting procedure because of the presence of an

\footnotetext{
1 http://basebe.obspm. fr

2 Shelyak Instruments, http://www . shelyak. com
} 


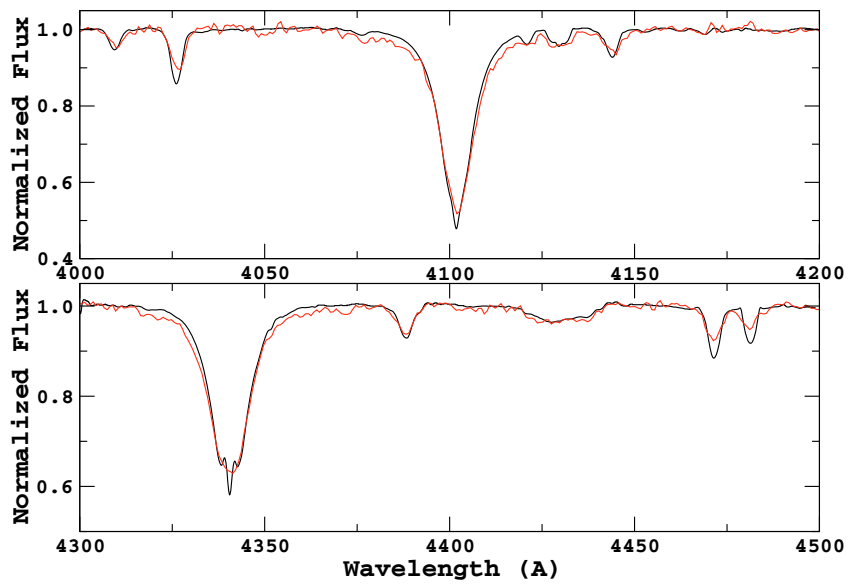

Fig. 2. Comparison between the low resolution spectrum used in F06 (red line) and the averaged FEROS spectrum from this paper (black line) with a resolution reduced to that of F06.

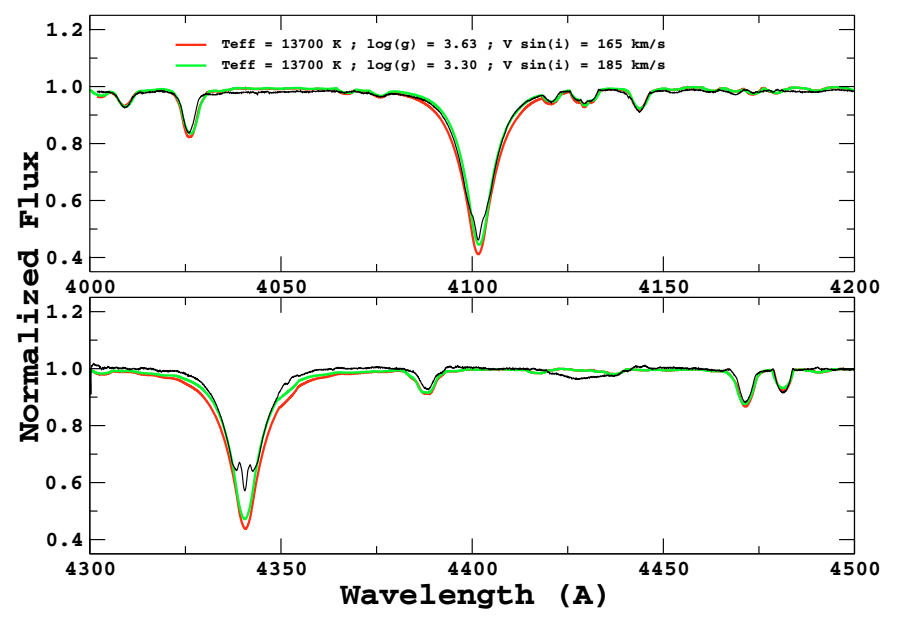

Fig. 3. Example of two synthetic spectral fits of the observations to determine the fundamental parameters using two sets of parameters.

interstellar band at $4425 \AA$. The average value of the parameters that we obtained are given in Table 2, with their uncertainties.

Error bars were estimated by performing multiple fits, selecting different lines and fixing one parameter $\left(T_{\text {eff }}\right.$ or $\left.\log g\right)$ at a time, while allowing the others to vary. The range of parameters that provide acceptable agreement with the observations infer the accuracy of the parameters (see e.g., Fig. 3).

For comparison, we also determined $v \sin i$ by computing the Fourier transform (see e.g., Royer et al. 2002) of the C II 4267 line profile (Fig. 4). This line was chosen because it appears to be unaffected by emission. The result is given in Table 2 and is coherent with that found by fitting the helium line profiles.

Assuming that $\frac{\Omega}{\Omega_{\mathrm{c}}}$ (where $\Omega$ and $\Omega_{\mathrm{c}}$ are the angular velocity and its critical value, respectively) ranges from 0.8 to 0.99 , we corrected the stellar parameters for gravitational darkening effects following F06 and using corrections computed in Frémat et al. (2005) to obtain an estimate of the stellar parameters of the non-rotating counterpart of the star as well as its true $v \sin i$ value. The results are listed in Table 3. We find in particular that the rotation frequency is $f_{\text {rot }}=0.75 \pm 0.32 \mathrm{c} \mathrm{d}^{-1}$.

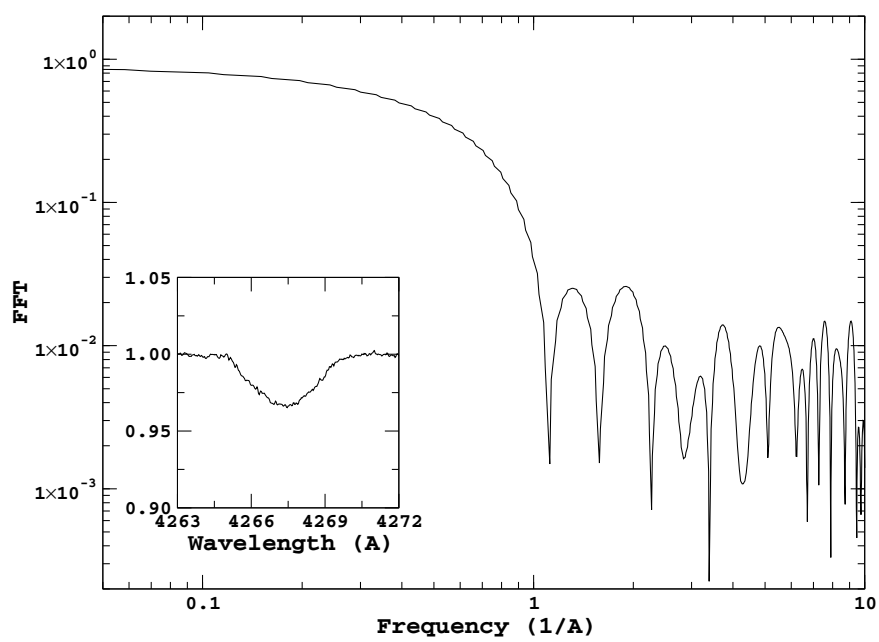

Fig. 4. Determination of the $v \sin i$ value with the Fourier technique.

\subsection{Disk emission}

The $\mathrm{H} \alpha, \mathrm{H} \beta$, and $\mathrm{H} \gamma$ lines exhibit strong emission in the profiles and $\mathrm{H} \delta$ shows an inverted winebottle shape. Following the atlas of Hanuschik et al. (1996), we classify the star on the basis of its $\mathrm{H} \alpha$ and $\mathrm{Fe}$ II 5316 line profiles and estimate the disk inclination.

The average profiles of the $\mathrm{H} \alpha, \mathrm{H} \beta$, and Fe II 5316 lines are displayed in Fig. 5. The line profiles exhibit two symmetrical emission peaks (class 1) with weak winebottle shoulders and $\mathrm{H} \alpha$ even exhibits a third peak in its red wing. Therefore, the star may be classified as L1 (inclinations of between 10 and 60 degrees) in the Hanuschik atlas. This is compatible with the value derived above from the spectral fitting of fundamental parameters (Table 3). We note, that the $V$-shaped central depression of the Fe II 5169 and 5316 profiles implies that the star should rather be classified as $\mathrm{H} 1$ (inclinations between 60 and 80 degrees). However, these lines are much less reliable for a disk classification.

Following Eq. (7) in Hummel \& Vrancken (1995), we determine the radius of the circumstellar disk from the distance between the averaged $V$ and $R$ peaks of $\mathrm{H} \alpha$. We find that $R_{\mathrm{d}}=(2 * 169 / 92.3)^{2}=13.4 R_{*}$.

The intensity of the emission in $\mathrm{H} \alpha$ compared to the continuum level, observed in the spectra retrieved from the BeSS database and obtained with FEROS and Narval during the CoRoT run, is plotted in Fig. 6. The BeSS datapoints exhibit a scatter caused by the lower signal-to-noise ratio of these spectra obtained with small telescopes. Intrinsic variations are, however, clearly observed in the FEROS and Narval data with an estimated period of $\sim 15$ days. The $V, R$, and $V / R$ quantities measured in the $\mathrm{H} \alpha$ line also exhibit variations but no clear periodicity.

\subsection{Variability}

Although HD 181231 is classified as constant in the Hipparcos catalogue (Perryman et al. 1997), it was re-classifed instead as variable on the basis of ground-based spectroscopic observations with a frequency at $0.67 \mathrm{c} \mathrm{d}^{-1}$ (Gutiérrez-Soto et al. 2007).

\subsection{Magnetic field}

We analysed the spectropolarimetric Stokes $V$ data obtained with Narval to search for the presence of a magnetic field. We 
Table 3. Parameters corrected for gravitational darkening effects at different $\frac{\Omega}{\Omega_{\mathrm{c}}}$ values.

\begin{tabular}{lllllllll}
\hline \hline$\frac{\Omega}{\Omega_{\mathrm{c}}}$ & $\begin{array}{l}T_{\text {eff }} \\
(\mathrm{K})\end{array}$ & $\log g$ & $\begin{array}{l}v \sin i \\
\left(\mathrm{~km} \mathrm{~s}^{-1}\right)(\mathrm{deg})\end{array}$ & $\begin{array}{l}R_{\mathrm{eq}} \\
\left(R_{\odot}\right)\end{array}$ & $\begin{array}{l}f_{\mathrm{rot}} \\
\left(\mathrm{c} \mathrm{d}^{-1}\right)\end{array}$ & $\begin{array}{l}\mathrm{M} \\
\left(M_{\odot}\right)\end{array}$ & $\begin{array}{l}\log \\
L / L_{\odot}\end{array}$ \\
\hline 0.80 & 14300 & 3.62 & 178 & $49 \pm 10$ & $6.5 \pm 2.5$ & $0.72 \pm 0.32$ & $4.4 \pm 1.3$ & 2.8 \\
0.90 & 14300 & 3.59 & 191 & $44 \pm 7$ & $7.2 \pm 2.8$ & $0.76 \pm 0.33$ & $4.4 \pm 1.3$ & 2.9 \\
0.95 & 14200 & 3.57 & 195 & $40 \pm 6$ & $7.8 \pm 2.9$ & $0.78 \pm 0.31$ & $4.5 \pm 1.4$ & 2.9 \\
0.99 & 14000 & 3.51 & 210 & $40 \pm 6$ & $8.9 \pm 3.4$ & $0.73 \pm 0.31$ & $4.5 \pm 1.4$ & 2.9 \\
\hline
\end{tabular}

The error bars in $T_{\text {eff }}$ are $\pm 1500 \mathrm{~K}$, in $\log g \pm 0.20$, in $v \sin i \pm 25 \mathrm{~km} \mathrm{~s}^{-1}$ and in $\log L / L_{\odot} \pm 0.5$.

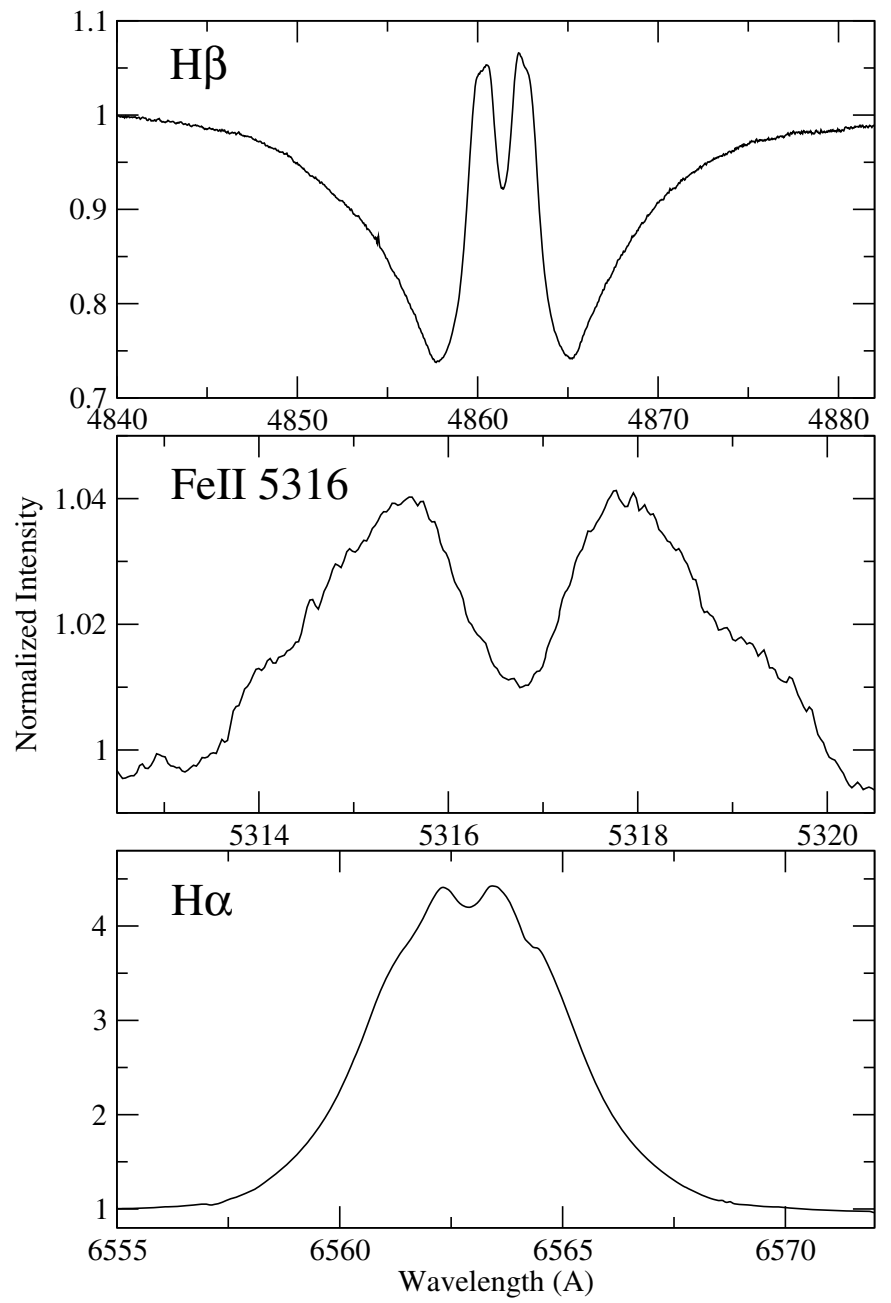

Fig. 5. Averaged line profiles of the $\mathrm{H} \alpha, \mathrm{H} \beta$, and $\mathrm{Fe}$ II 5316 lines from FEROS data.

used the Least Square Deconvolution (LSD) technique developed by Donati et al. (1997). The list of lines that we used was compiled from a synthetic spectrum template for a star with $T_{\text {eff }}=15000 \mathrm{~K}$ and $\log g=3.5$, from which we removed all lines contaminated by circumstellar emission and lines invisible in the spectrum of HD 181231. The final list contains 139 lines.

The 18 measurements of the longitudinal magnetic field of HD 181231 infer values compatible with 0 with typical error bars of the order of $650 \mathrm{G}$. We thus find no evidence of a magnetic field in this star at the detection level we reached, i.e., we can define an upper limit to $B_{\text {long }}$ equal to $\sim 650 \mathrm{G}$ in our observations.

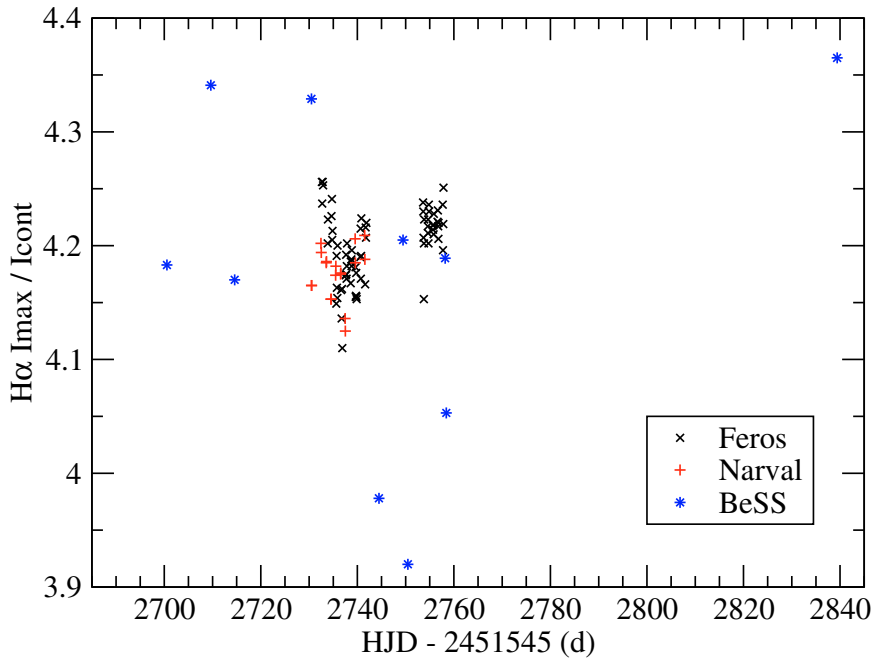

Fig. 6. Variation in the emission intensity in the $\mathrm{H} \alpha$ line as observed during the CoRoT run with the FEROS and Narval spectrographs and by amateur astronomers C. Buil, J. Ribeiro, and O. Thizy as a contribution to the BeSS database.

\section{Time-series analysis}

\subsection{CoRoT photometric data}

CoRoT data were analysed using several Fourier techniques: ClEAN-NG, PASPER, and TISAFT, all developed within the CoRoT Be team. TISAFT uses a statistical Fisher test to decide which of the peaks in the power spectrum correspond to significant signal. CLEAN-NG uses a stopping criterion based on the relative rate $r$ of increase in the total power in the clean components from one iteration to the next one. We used $r=0.0001$ and an oversampling of 16 . We note that PASPER collects peaks of highest amplitudes until it finds one peak that does not fulfil the empirical criterion $S / N>4$. Since the noise decreases with frequency, it can thus miss peaks at high frequencies with $S / N>4$ but low amplitudes. For a detailed description of these methods, we refer to Gutiérrez-Soto et al. (2009).

Frequencies found with these 3 methods, their amplitude and phase, as well as the interpretation of each frequency in terms of stellar signal or combinations, but excluding instrumental frequencies such as the orbital frequency of the satellite, are given in Table 5. The amplitudes and phases, and their errors, obtained for each set of frequencies with these three methods were recalculated with AMPHI to achieve greater accuracy and homogeneity and are also given in Table 5. For a complete set of fixed frequencies, this program calculates the amplitudes and phases to obtain the best fit to the light curve (see Gutiérrez-Soto et al. 2009).

The 3 methods were applied to the CoRoT light curve with both gaps and interpolated points (see Sect. 2) and yielded 


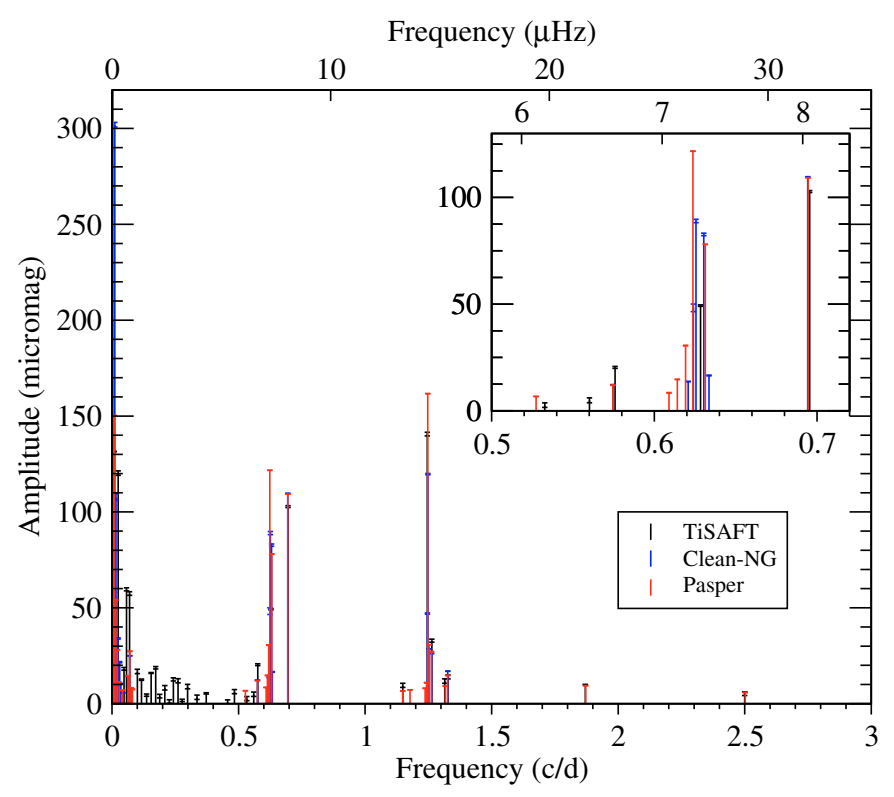

Fig. 7. Power spectrum of all significant frequencies detected by TISAFT, ClEAN-NG, and PASPER. A zoom at the main frequencies is shown in the top right panel, with the same axis as in the main panel.

similar results. The interpolation, however, allows us to easily suppress peaks produced by the observing window (see Gutiérrez-Soto et al. 2009). The minimum detectable frequency is $1 / T=0.0064 \mathrm{c} \mathrm{d}^{-1}$, i.e., $0.075 \mu \mathrm{Hz}$, where $T$ is the length of the run. Following Kallinger et al. (2008), the frequency uncertainty of close peaks is $\Delta f=1 /(4 T)=0.0016 \mathrm{c} \mathrm{d}^{-1}$, i.e., $0.019 \mu \mathrm{Hz}$. A power spectrum of all significant frequencies obtained with the three methods is shown in Fig. 7.

The main frequencies were found with all three methods. We note however that TISAFT detects a frequency at $0.6284 \mathrm{c} \mathrm{d}^{-1}$ that differs slightly from the one at $0.624 \mathrm{~cd}^{-1}$ obtained with the other two methods, and of lower amplitude. In the case of CLEAN-NG, the frequency at $0.624 \mathrm{c} \mathrm{d}^{-1}$ is divided into two close peaks and the sum of the two amplitudes $(1.372 \mathrm{mmag})$ should be considered as the amplitude for this frequency.

Moreover, TISAFT detects several frequencies between 0.1 and $0.4 \mathrm{~cd}^{-1}$, which the other two methods do not detect. These frequencies could be related to the sync function of the low frequencies and should be considered with care.

A plot of the CoRoT light curve folded in phase with the two detected frequencies of the highest amplitudes $\left(f 1=0.695 \mathrm{c} \mathrm{d}^{-1}\right.$ and $\left.f 2=0.624 \mathrm{c} \mathrm{d}^{-1}\right)$ is shown in Fig. 8. A phase-binned curve is superimposed on each panel, as well as a sinusoidal fit. We see that $f 1$ corresponds to a single sinusoidal wave, while $f 2$ corresponds to a double sinusoidal wave. This explains why we find a high amplitude frequency peak at the harmonic value $2 *$ $f 2$.

In addition, we performed a time-frequency analysis of the CoRoT data using a sliding window of various lengths, shifted day by day, and various oversampling values. This method is described in detail in Huat et al. (2009). We either searched for frequencies with CLEAN-NG or PASPER in each sub-sample, or imposed the main frequencies detected over the entire run. We then recalculated the amplitudes and phases with AMPHI. We found similar results regardless of the window size and with or without imposing the frequency values.

A 3D plot of all frequencies detected with the timefrequency method, for a window of 30 days and an oversampling

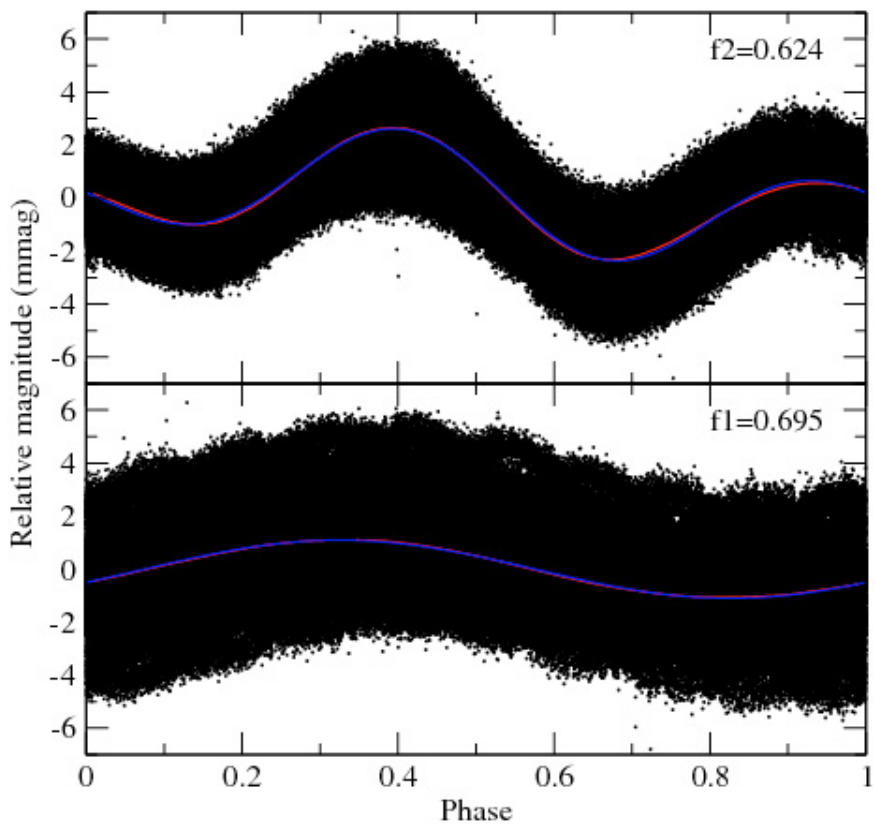

Fig. 8. CoRoT light curve folded with the two main frequencies detected. A phase-binned curve (red) and a sinusoidal fit (blue) as a single (for $f 1$ ) or double wave (for $f 2$ ) are superimposed in each panel.

of 16, is shown in Fig. 9. When viewed from the top (bottom panel), this plot corresponds to a periodogram. In this figure, the variation in amplitude with time is clearly evident, in particular, we observe a modulation of the amplitude of the signal at $\sim 60$ and $\sim 120$ days. This long $\sim 120$-d modulation corresponds to the 116-d amplitude variation observed in the CoRoT light curve (Fig. 1) and to the frequency $\sim 0.00848 \mathrm{c} \mathrm{d}^{-1}$ detected in the light-curve analysis. Variations on shorter timescales are also observed.

A similar analysis with a method based on the Morlet wavelet (Baudin et al. 1994, 1996) was performed (see Fig. 11). The Morlet wavelet provides a superior trade-off between frequency resolution and time resolution than the above method. Nevertheless, it yields similar results.

\subsection{Ground-based spectroscopic data}

A time series analysis is performed using Fourier Transform and least squares fitting methods, as in Floquet et al. (2002) and Neiner et al. (2005) with the FEROS data. These methods are applied to each wavelength bin of the line-profile time series to study the line profile variations (lpv) of the He I 4026, 4143, 4387, 4471, 4713, 4922, 6678, Mg II 4481, Si II 4128, 4130, 5056, $\mathrm{C}$ II $4267, \mathrm{H} \alpha$ and $\mathrm{H} \beta$ lines. The minimum detectable frequency is $1 / T=0.04 \mathrm{c} \mathrm{d}^{-1}$, i.e., $0.46 \mu \mathrm{Hz}$, where $T$ is the length of the FEROS run. The error bar of the frequencies is $\Delta f=1 /(4 T)=0.01 \mathrm{c} \mathrm{d}^{-1}$, i.e., $0.12 \mu \mathrm{Hz}$.

The frequency $0.69 \mathrm{~cd}^{-1}$ is detected in the $\mathrm{lpv}$ of the $\mathrm{Mg}$ II 4481 and He I 4471 lines. This frequency corresponds to $f 1 \mathrm{de}-$ tected in the CoRoT data. The power and phase distributions of this frequency are displayed in Fig. 10 for the two lines. We note that the power is higher in the wings than in the centre of the lines. In terms of non-radial pulsations, an initial guess of the pulsation parameters can be obtained from the Intensity Period Search method (Telting \& Schrijvers 1997). The blue-tored phase difference found for a frequency is an indicator of the typical degree $\ell$. From the phase difference of the variations with 

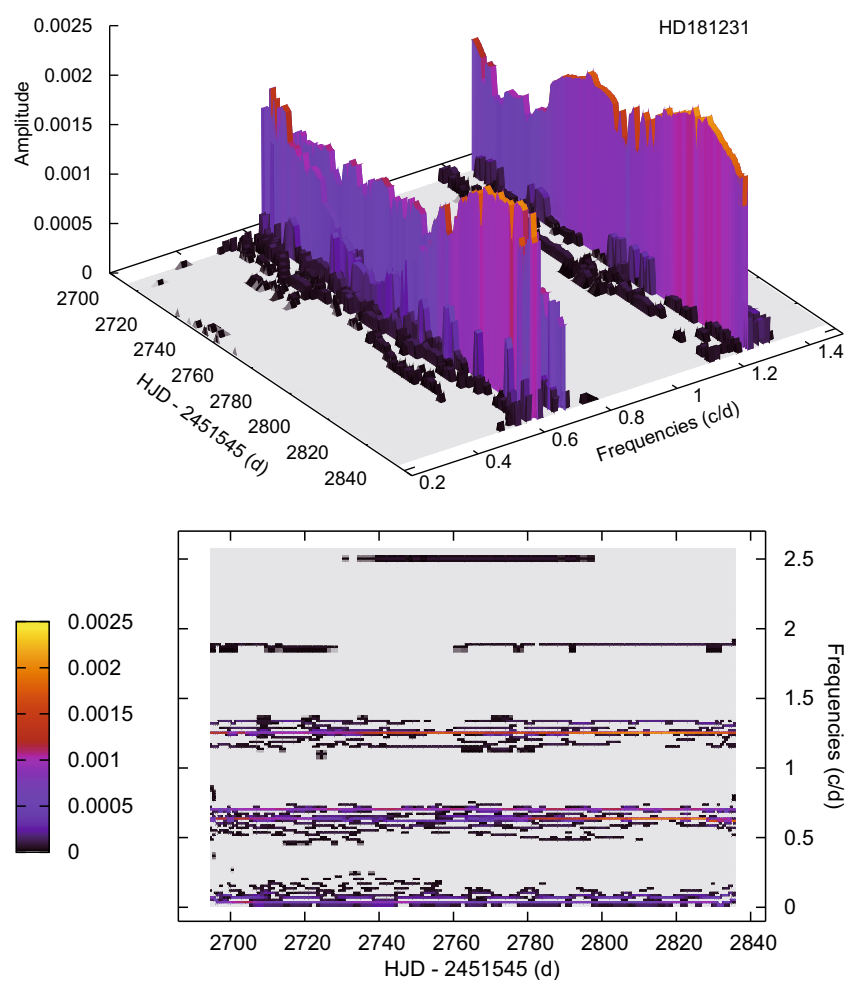

Fig. 9. 3D view of the frequencies determined using a sliding window of 30 days. The colours indicate the amplitude of the frequency peaks. The bottom panel shows the same plot viewed from the top.

Table 4. Frequencies (in $\mathrm{c} \mathrm{d}^{-1}$ ) deduced from the time-series analysis of various parameters (Cols. 3 to 7 ) of all studied lines listed in Col. 1 with the FEROS data. The symbol "-" means that a frequency search was performed but no significant frequency was found. The second column $V_{\text {rad }}$ gives the value of the average radial velocity of each line (in $\mathrm{km} \mathrm{s}^{-1}$ ). In this column, the symbol "-" means that we did not calculate $V_{\text {rad }}$ due to the presence of emission or blended lines.

\begin{tabular}{lllllll}
\hline \hline Line & $\begin{array}{l}V_{\mathrm{rad}} \\
\mathrm{km} \mathrm{s}^{-1}\end{array}$ & $\begin{array}{l}l p v \\
\mathrm{c} \mathrm{d}^{-1}\end{array}$ & $\begin{array}{l}\mathrm{RV} \\
\mathrm{cd}^{-1}\end{array}$ & $\begin{array}{l}\mathrm{EW} \\
\mathrm{c} \mathrm{d}^{-1}\end{array}$ & $\begin{array}{l}\mathrm{CD} \\
\mathrm{c} \mathrm{d}^{-1}\end{array}$ & $\begin{array}{l}F W H M \\
\mathrm{c} \mathrm{d}^{-1}\end{array}$ \\
\hline He I 4026 & -5 & 1.42 & & & & \\
Si II 4128 & - & 0.30 & & & & \\
He I 4143 & 13 & 1.24 & & & & \\
C II 4267 & 6 & 1.25 & & & & - \\
He I 4387 & 18 & 0.36 & - & & - & - \\
He I 4471 & 4 & 0.69 & 0.69 & 1.38 & 0.69 & - \\
Mg II 4481 & 14 & 0.69 & 0.69 & - & 0.69 & 0.69 \\
He I 4713 & 14 & 0.88 & & & & \\
H $\beta$ & - & 1.26 & & & & \\
He I 4922 & - & 0.64 & & & & \\
Fe II 5316 & - & 0.35 & & & & \\
He I 6678 & 16 & 0.30 & & & & \\
H $\alpha$ & - & 0.67 & & & & \\
\hline
\end{tabular}

the frequency $0.69 \mathrm{~cd}^{-1}(\Delta \phi \sim 3)$, we thus estimate the $\ell$-degree of the mode to be $\sim 3$.

We note that the spectra are contaminated with Fe II lines in emission, because of the circumstellar disk. This is probably why we only find a coherent phase in the $\mathrm{Mg}$ II 4481 and $\mathrm{He}$ I 4471 lines for $f=0.69 \mathrm{c} \mathrm{d}^{-1}$. Greyscale dynamical spectra of the Mg II 4481 and He I 4471 lines folded in phase with the frequency $0.69 \mathrm{c} \mathrm{d}^{-1}$ were calculated. However, they do not show any clear pattern.

Other detected frequencies for the FEROS data, given in Table 4, have incoherent phase distributions. Most of them are

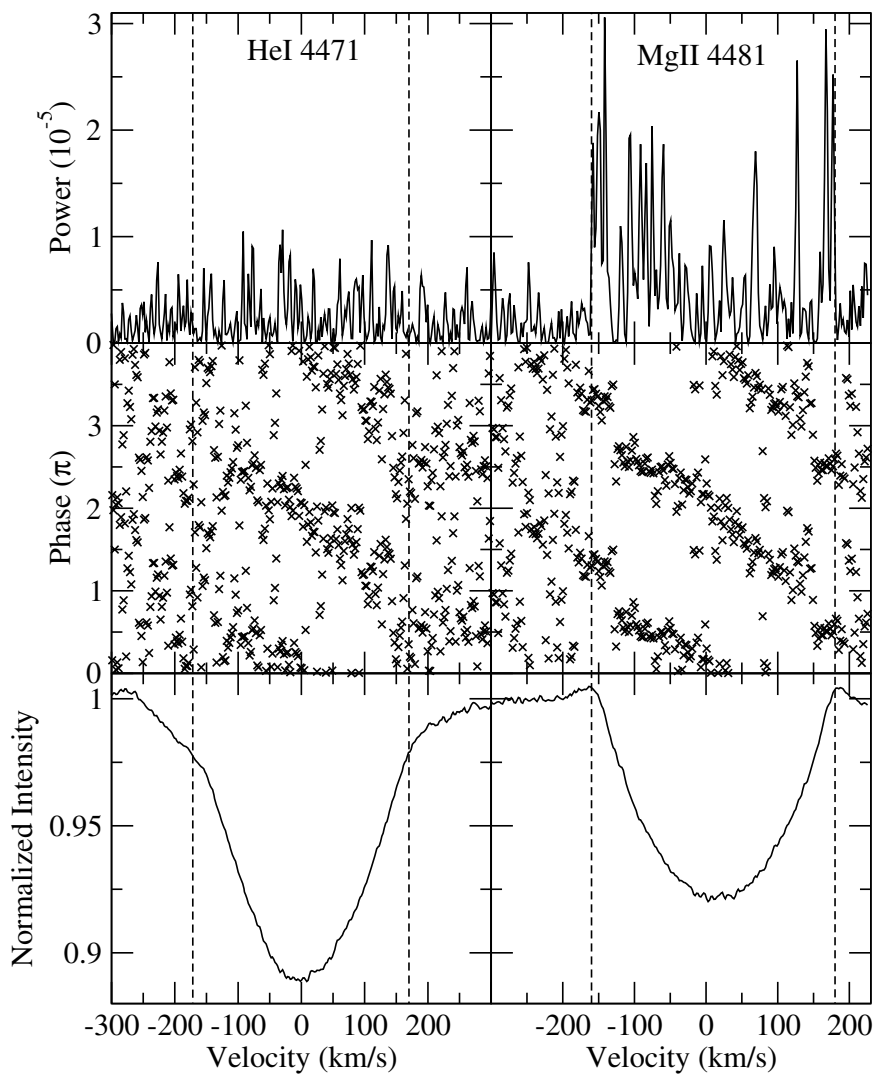

Fig. 10. Power and phase distributions of the frequency at $0.69 \mathrm{~cd}^{-1}$ for the He I 4471 and $\mathrm{Mg}$ II 4481 lines and their averaged line profile. Dashed lines indicate the $\pm v \sin i$ values.

probably pseudo-periods or artifacts in the observations. We note however that a frequency at $\sim 1.25 \mathrm{c} \mathrm{d}^{-1}$ is detected in the $\mathrm{He}$ I 4143, C II 4267, and $\mathrm{H} \beta$ lines, which corresponds to the harmonics $2 * f 2$ detected in the CoRoT data. A frequency at $0.64 \mathrm{c} \mathrm{d}^{-1}$, i.e., close to $f 2$, is also detected in the He I 4922 line.

The radial velocity (RV) of the line centroid, the central depth (CD), the full-width at half-maximum ( $F W H M)$, and the equivalent width $(\mathrm{EW})$ of some of the lines are also studied in detail for each line profile with the standard Fourier techniques. A non-linear multi-parameter fitting code (Vaníček 1971) was used to search for frequencies in these spectral parameters.

We found again the frequency $0.69 \mathrm{c} \mathrm{d}^{-1}$ in the variations of the RV, CD, and FWHM of the Mg II 4481, and in the RV and $\mathrm{CD}$ of the He I 4471 lines. Twice this frequency, $1.38 \mathrm{c} \mathrm{d}^{-1}$, is detected in the EW of the He I 4471 line. A clear frequency is detected in neither the other line quantities of these two lines, nor the He I 4387 line.

The phase diagram with $f 1=0.695 \mathrm{c} \mathrm{d}^{-1}$ for the EW of the Mg II 4481 line is shown in Fig. 12. The binned and scaled CoRoT light curve folded with this frequency has been superimposed. We note that the radial velocity maximum occurs about a quarter of a period $(\Delta \Phi=0.28)$ after maximum light.

The small number of Narval spectra does not allow us to perform a complete analysis of the lpv or spectral quantities for those data. Nevertheless, a frequency at $0.67 \mathrm{c} \mathrm{d}^{-1}$ was detected in the lpv for the Mg II 4481 and He I 4471 lines, but with no coherent phase diagram, probably because of the uncertainty in the determination of the sinusoidal function. However, the detected frequency is consistent with the frequency at $0.69 \mathrm{c} \mathrm{d}^{-1}$ detected in the FEROS data. 

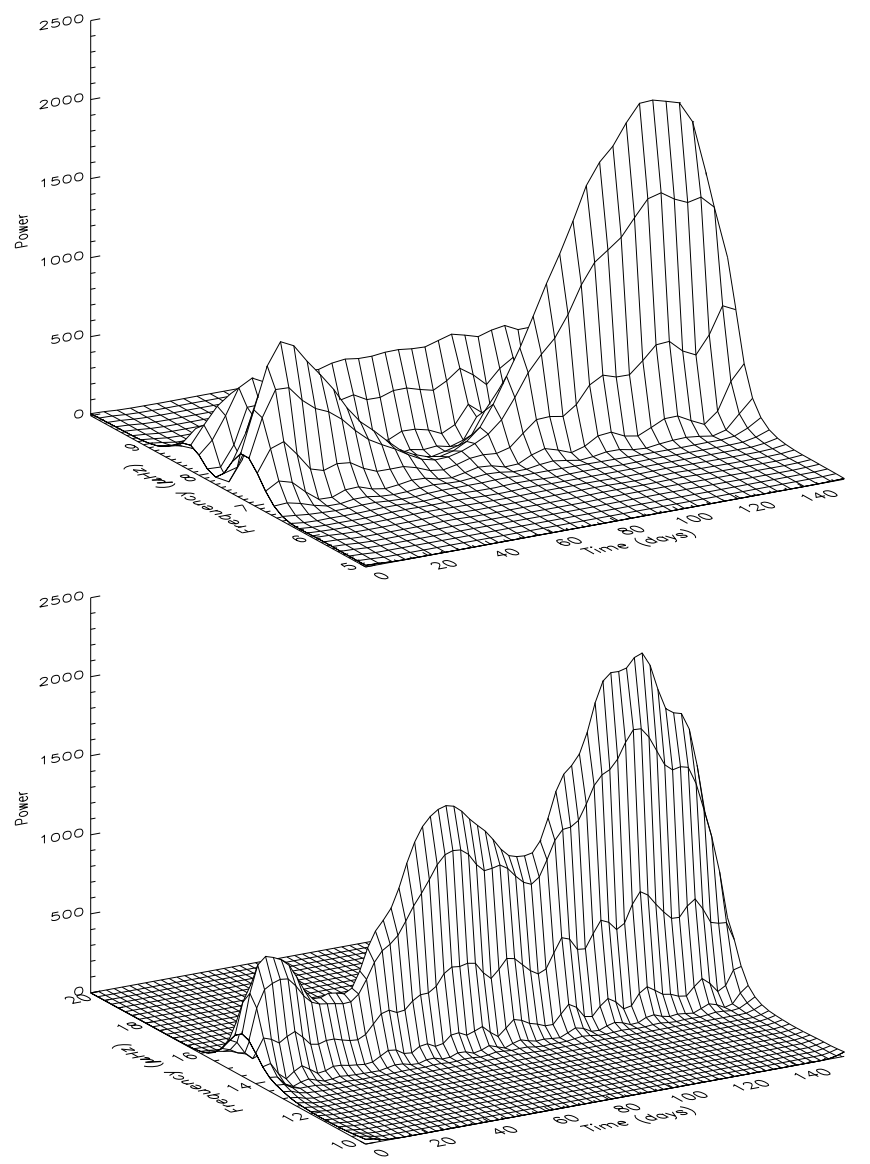

Fig. 11. Time-frequency diagrams centred in frequency on the signal around 0.624 and $0.695 \mathrm{c} \mathrm{d}^{-1}\left(7.2\right.$ and $8.0 \mu \mathrm{Hz}$, top panel) and $1.24 \mathrm{c} \mathrm{d}^{-1}$ $(14.4 \mu \mathrm{Hz}$, bottom panel), showing the variation in power versus time and frequency. The power is normalized by the mean power of the local noise.

\section{Discussion}

In the following sections, we discuss the results obtained in this work for the Be star HD 181231 in the light of pulsations, rotation, and beating effects.

\subsection{Pulsations and rotation}

We have detected at least 10 significant independent frequencies of variations in the CoRoT light curve of HD 181231. This number of detected frequencies may seem small compared to the e.g., $\sim 60$ frequencies detected for HD 163868 using a few weeks of MOST observations (Walker et al. 2005), while we have observed HD 181231 during 5 months. However, we have cleaned our list of 54 detected frequencies from all combination frequencies and harmonics. Therefore, we consider here only independent true stellar frequencies. We note in addition that, in hot stars, each detected frequency provides information about a different layer of the star. Therefore, seismic modelling can be very powerful for those objects even when only a few frequencies are detected.

The frequency peak with the second highest amplitude $(1.1 \mathrm{mmag})$ is detected at $f 1=0.695 \mathrm{c} \mathrm{d}^{-1}(8.04 \mu \mathrm{Hz})$ and corresponds to an isolated peak in the power spectrum. This frequency is compatible with the rotation frequency $\left(f_{\text {rot }}=\right.$ $\left.0.75 \pm 0.32 \mathrm{~cd}^{-1}\right)$ determined from spectral fitting. However, this frequency is also detected in spectroscopy in the line-profile

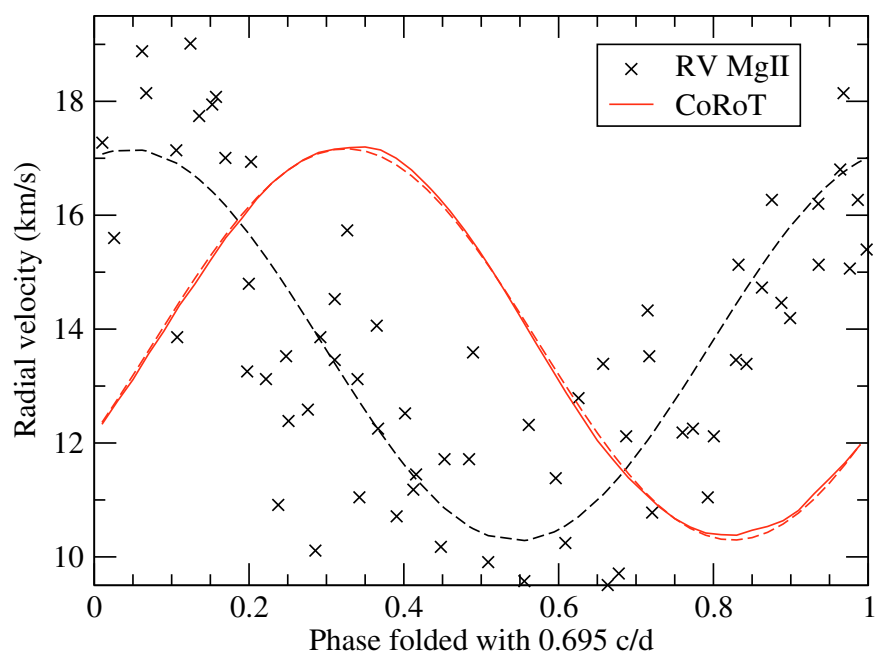

Fig. 12. Phase diagram of the RV variations with the frequency $0.695 \mathrm{c} \mathrm{d}^{-1}$ for the Mg II 4481 line (black crosses). The CoRoT light curve folded with this frequency, binned, and scaled to be comparable to the RV measurements has been superimposed (red solid line). A sine fit of both datasets is superimposed (dashed lines). A phase shift between the 2 curves of 0.28 phase can be noticed.

variations as well as in spectral quantities, and the amplitude and phase distributions observed in the line-profile variations with this frequency (Fig. 10) are typical of non-radial pulsations with a mode degree $\ell \sim 3$. The CoRoT light curve folded in phase with this frequency is sinusoidal (Fig. 8) and no harmonic of this frequency is detected even though it corresponds to a high amplitude peak, which is also an indication of pulsation rather than rotation. A phase shift of 0.28 in a period is observed between the variation with this frequency in the radial velocity measured in spectral lines and the one observed in the CoRoT light curve (Fig. 12). In terms of non-radial pulsations this phase shift can be explained by non-adiabatic effects (see Dupret et al. 2003), time-variable frequencies, or resonant phase-locking. We thus conclude that the frequency $f 1=0.695 \mathrm{c} \mathrm{d}^{-1}$ is due to non-radial pulsations with a typical $\ell \sim 3$. We note that, in rapidly rotating stars, g-modes with $\ell$ values of 3 or more can be detected, even if lower degree modes (e.g. $\ell=0$ ) are not detected, because of the coupling between modes (see Daszyńska-Daszkiewicz et al. 2002).

The frequency peak with the highest amplitude $(1.3 \mathrm{mmag})$ is detected at $f 2=0.624 \mathrm{~cd}^{-1}(7.22 \mu \mathrm{Hz})$. Several other frequency peaks are situated just below this frequency, so that it constitutes an entire group of close frequencies. This group includes in particular a frequency at $f 6=0.575 \mathrm{c} \mathrm{d}^{-1}(6.65 \mu \mathrm{Hz})$. For rapid rotators such as HD 181231, groups of close frequencies are expected from modes of non-radial pulsations with different $\ell$ values but the same azimuthal order $m$, since the rotational splitting is of the order of $f_{\text {rot }}$. The second, third, and fourth harmonics of $f 2$ are also detected. We note that $2 * f 2$ has an even stronger amplitude than $f 2$ itself. The CoRoT light curve folded in phase with this frequency is a double sinusoidal wave (Fig. 8). Harmonics can appear in photometry either if the main frequency corresponds to the rotation frequency or if it corresponds to a pulsation frequency with $\ell=0$ or of a high amplitude. In this case, $f 2$ is compatible with the rotation frequency ( $\left.f_{\text {rot }}=0.75 \pm 0.32 \mathrm{c} \mathrm{d}^{-1}\right)$ determined from spectral fitting, but since the amplitude of this frequency peak is high it could also be attributed to pulsations. Since $f 2$ has not been detected by our 
analysis of the available spectroscopy, we cannot draw firm conclusions on this matter. Seismic modelling is therefore required.

We note that the results for $f 1$ and $f 2$ show the importance of acquiring spectroscopic observations simultaneously with space photometric observations.

Many combinations of frequencies are also detected as significant peaks in the Fourier analysis of the CoRoT light curve, in particular those resulting from the difference between $f 2$ and $f 1$ or the other peaks in the group around $f 1$. Frequencies detected close to high amplitude peaks could also be caused by changes in the amplitude or phase of the main frequencies.

\subsection{Beating effects}

Two longer-term variations are observed in the CoRoT data at $\sim 14$ days and $\sim 116$ days (see Fig. 1).

The $\sim 14-d$ period is clearly visible in the CoRoT light curve (Fig. 1). A frequency for this signal of $f=0.07 \mathrm{c} \mathrm{d}^{-1}$, i.e., $0.8 \mu \mathrm{Hz}$, is detected in the analysis of the CoRoT light curve and corresponds to the difference between the two main frequency peaks at $f 1=0.695$ and $f 2=0.624 \mathrm{c} \mathrm{d}^{-1}$. Therefore, this can be clearly identified as a beating phenomenon between the two main frequency of variations.

This frequency is also detected in our spectroscopic analysis in the variation of the intensity of $\mathrm{H} \alpha$ emission (Fig. 6). This quantity provides direct insight into the circumstellar environnement. Therefore, it seems that the beating effect influences the circumstellar environment. A correlation between pulsation properties and Be outbursts was observed in the star HD 49330 (Huat et al. 2009). We note also that the beating frequency corresponds to a tenth of $f 1$, which is probably a coincidence.

The 116-d period is visible in the CoRoT light curve (Fig. 1) as well as in the 3D representation shown in Fig. 9. The frequency of this signal ( $f=0.00848 \mathrm{c} \mathrm{d}^{-1}$, i.e., $\left.0.098 \mu \mathrm{Hz}\right)$ is detected in the analysis of the CoRoT light curve and corresponds to the difference between $f 2=0.624 \mathrm{c} \mathrm{d}^{-1}$ and lower amplitude peaks around $f 1$ namely $f 5$ and $f 9$. Frequencies of different $\ell$ degree values but identical azimuthal order $m$ are expected to be close frequencies for fast rotators. Moreover, a coupling between frequencies as close as those and with a $\ell$ difference of 2 is expected (Daszyńska-Daszkiewicz et al. 2002; Soufi et al. 1998). Alternatively, according to seismic modelling performed by Saio and collaborators (e.g. Cameron et al. 2008), one could consider $f=0.00848 \mathrm{c} \mathrm{d}^{-1}$ to be a pulsation mode with $m=0$. Hence, $f 5$ and $f 9$ would then be the combination frequencies of this zonal mode and $f 2$.

\section{Conclusions}

Thanks to the long duration and high photometric accuracy of the CoRoT observations of the Be star HD 181231, we have detected a large number of variation frequencies. This list of 54 detected frequencies can be reduced to a set of at least 10 independant frequencies corresponding to the intrinsic variation frequencies of the star. For each frequency, we have determined the average amplitude and phase during the observing run. One of these frequencies $(f 1)$ is also detected by our analysis of the available spectroscopic data, which allows us to identify it as a pulsation mode with a typical $\ell$ value of $\sim 3$. Other lower amplitude peaks are probably also caused by non-radial pulsation modes. A high amplitude frequency peak $(f 2)$ could be attributed to either rotation or pulsations.

In addition, we have shown that a beating effect occurs between the two strongest frequencies ( $f 1$ and $f 2$ ) with a period of about 14 days. A longer-term beating (116 days) is also observed.

By analysing the spectroscopic data acquired for this work, we have also determined more precisely the fundamental parameters of the star. These results and the seismic results presented above will allow us to calculate models of the interior of this star. This will be the subject of a forthcoming paper.

Acknowledgements. The CoRoT space mission, launched on December 27th 2006, has been developed and is operated by CNES, with the contribution of Austria, Belgium, Brasil, ESA, Germany and Spain. We wish to thank the CoRoT team for the acquisition and reduction of the CoRoT data. This research is based on data obtained at the Télescope Bernard Lyot (Pic du Midi). The FEROS data have been obtained as part of the ESO Large Programme LP178.D0361 (PI: Poretti). This work has made use of the BeSS database, operated at GEPI, Observatoire de Meudon, France: http : //basebe .obspm. fr. We thank the BeSS observer B. Heathcote.

\section{References}

Auvergne, M., Bodin, P., Boisnard, L. et al. 2009, A\&A, 506, 411

Baudin, F., Gabriel, A., \& Gibert, D. 1994, A\&A, 285, L29

Baudin, F., Gabriel, A., Gibert, D., Palle, P. L., \& Regulo, C. 1996, A\&A, 311, 1024

Cameron, C., Saio, H., Kuschnig, R., et al. 2008, ApJ, 685, 489

Cranmer, S. R. 2005, ApJ, 634, 585

Daszyńska-Daszkiewicz, J., Dziembowski, W. A., Pamyatnykh, A. A., \& Goupil, M.-J. 2002, A\&A, 392, 151

Donati, J.-F., Semel, M., Carter, B. D., Rees, D. E., \& Collier Cameron, A. 1997, MNRAS, 291, 658

Dupret, M.-A., De Ridder, J., De Cat, P., et al. 2003, A\&A, 398, 677 Dziembowski, W. A., \& Pamiatnykh, A. A. 1993, MNRAS, 262, 204 Dziembowski, W. A., Moskalik, P., \& Pamyatnykh, A. A. 1993, MNRAS, 265, 588

Floquet, M., Neiner, C., Janot-Pacheco, E., et al. 2002, A\&A, 394, 137

Frémat, Y., Zorec, J., Hubert, A.-M., et al. 2005, A\&A, 440, 305

Frémat, Y., Neiner, C., Hubert, A.-M., et al. 2006, A\&A, 451, 1053 (F06)

Gutiérrez-Soto, J., Fabregat, J., Suso, J., et al. 2007, A\&A, 476, 927

Gutiérrez-Soto, J., Floquet, M., Samadi, R., et al. 2009, A\&A, 506, 133

Hanuschik, R. W., Hummel, W., Sutorius, E., Dietle, O., \& Thimm, G. 1996, A\&AS, 116, 309

Huat, A.-L., Hubert, A.-M.., Baudin, F., et al. 2009, A\&A, 506, 95

Hubert, A. M., \& Floquet, M. 1998, A\&A, 335, 565

Hummel, W., \& Vrancken, M. 1995, A\&A, 302, 751

Kallinger, T., Reegen, P., \& Weiss, W. W. 2008, A\&A, 481, 571

Kozok, J. R. 1985, A\&AS, 62, 7

Martayan, C., Frémat, Y., Hubert, A.-M., et al. 2006, A\&A, 452, 273

Martayan, C., Frémat, Y., Hubert, A.-M., et al. 2007, A\&A, 462, 683

Neiner, C., Floquet, M., Hubert, A. M., et al. 2005, A\&A, 437, 257

Perryman, M. A. C., Lindegren, L., Kovalevsky, J., et al. 1997, A\&A, 323, L49

Porter, J. M., \& Rivinius, T. 2003, PASP, 115, 1153

Rainer, M. 2003, Ph.D. Thesis, Universitá degli Studi di Milano

Royer, F., Gerbaldi, M., Faraggiana, R., et al. 2002, A\&A, 381, 105

Samadi, R., Fialho, F., Costa, J. E. S., et al. 2006, ESA SP 1306, 317, corrected in [arXiv:astro-ph/0703354]

Soufi, F., Goupil, M. J., \& Dziembowski, W. A. 1998, A\&A, 334, 911

Telting, J. H., \& Schrijvers, C. 1997, A\&A, 317, 723

Uytterhoeven, K., Mathias, P., Poretti, E., et al. 2008, A\&A, 489, 1213

Vaníček, P. 1971, Ap\&SS, 12, 10

Walker, G. A. H., Kuschnig, R., Matthews, J. M., et al. 2005, ApJ, 635, L77 